following the introduction of CPIPS. Studies are needed to report holistic outcomes, including chronic medical conditions and the mental health to continue developing a service to support chronic, complex, and life-long conditions.

\section{G165 USING VIDEO VIGNETTES AND DOCTORS' RESPONSES TO ASSESS THE EFFICACY OF PAEDIATRIC SIMULATION}

${ }^{1} \mathrm{M}$ Malley, ${ }^{2} \mathrm{M}$ Ko, ${ }^{3} \mathrm{M}$ Monaghan. ${ }^{1}$ West Middlesex Hospital, London, UK; ${ }^{2} \mathrm{FCransley}$ Hospice, Kettering, UK; ${ }^{3}$ Epsom General Hospital, London, UK

10.1136/archdischild-2018-rcpch. 161

Aims Paediatric simulation is an established part of medical student training. However there remain many ways to assess its efficacy. We aimed to assess the efficacy of a simulation programme using video-based clinical vignettes and comparing student responses to established paediatric doctors.

Method We created a paediatric simulation programme for 87 fifth year medical students. The programme involved two-hour theory session followed by a two-hour high fidelity simulation in groups of five.

Twelve clinical vignettes were created based on the videos from 'Spotting The Sick Child' online resource (with permission). The students stated how sick they thought the child was for each scenario on a scale of 1 to 10 , where 10 was 'immediate PICU admission' and 1 was 'immediate discharge'. This quiz was carried out pre- and post-course. Twenty post$\mathrm{MRCPCH}$ paediatricians carried out the same quiz. The average of their scores was considered the 'gold standard'. The deviation between the paediatrician and student scores was calculated both pre- and post-programme. Students also rated their confidence level in assessing and managing the cases before and after the programme.

Results Overall, students' responses were significantly closer to the doctors' mean post session $(\mathrm{p}=0.0048)$. This was particularly pronounced in respiratory distress, dehydration and DKA scenarios. The average value of students' confidence level for assessing paediatric emergencies pre- and post-simulation were $2.91 / 5$ and $3.58 / 5$, respectively $(\mathrm{p}<0.001)$. Similarly their reported confidence in managing the emergencies was $2.36 / 5$ pre-session and 3.36/5 post-session $(\mathrm{p}<0.000005)$.

Conclusion Video vignettes provided a novel approach to assessing the efficacy of a paediatric simulation programme. Students' responses more closely correlated with more experienced specialist physicians after the session compared to presession. The convergence of the paediatricians' and students' scores post-session was validated by the students' self-reported increased confidence level in assessing and managing the scenarios post-session. This supports the utility of this method for assessing the clinical educational efficacy of paediatric simulation programmes.

\section{G166 USEFULLNESS OF SOCIAL EMOTIONAL AND ADAPTIVE BEHAVIOUR AS PART OF TWO YEAR NEURODEVELOPMENTAL ASSESSMENTS}

${ }^{1} \mathrm{D}$ Metheniti, ' $\mathrm{M}$ Parmar, ${ }^{1} \mathrm{P}$ Southernwood, ${ }^{2} \mathrm{~S}$ Beasley, ${ }^{1} \mathrm{~N}$ Merchant. ${ }^{1}$ Paediatrics and Neonatology, West Hertfordshire NHS trust, Watford, UK; ${ }^{2}$ Peace Children's Centre Department of Physiotherapy, West Hertfordshire NHS Trust, Watford, UK
Introduction Neurodevelopmental outcome assessment provides basis for benchmarking and early intervention. Bayleys scale of infant development (BSID) is often used in 2 year assessments of high-risk infants, however there are time constraints in completing these assessments. Social, emotional and adaptive processes like self regulation and executive function which are increasingly being shown to predict later life and behaviour outcomes are often not captured in these assessments.

Aim To study if completion of social emotional and adaptive questionnaire of BSIDIII will be useful as part of the 2 year assessments.

Methods Social-emotional/adaptive parental questionnaire was given for all preterms born $<30$ weeks at the 2 year neurodevelopmental clinic. Demographic and infant data was collated along with the composite scores of the cognitive, language and motor, social emotional and adaptive scales of BSIDIII. Moderate delay was defined as composite score of 70-84 and severe delay as $<70$. Data was analysed using Microsoft Excel and STATA.

Results 19/48 (40\%) who returned the questionnaire had 2 year assessments at a mean age of 25 months. Table 1 shows the mean composite scores of different scales. There was significant correlation with general adaptive composite (GAC) and motor composite $(p=0.04,95 \% \mathrm{CI}-1.29$ to -0.33). A significant correlation was also seen with language composite and social adaptive domain $(p=0.042,95 \%$ CI -0.7 and -0.15$)$. There was no correlation seen with social emotional scale and motor/language/cognitive scales.

Abstract G166 Table 1 Mean composite scores from BSID subscales (GAC-general adaptive composite, CON-conceptual adaptive domain, SO- Social adaptive domain, PR-Practical adaptive domain)

\begin{tabular}{lllllllll}
\hline & Cognitive & Language & Motor & SE & GAC & CON & SO & PR \\
\hline Mean & 95.6 & 96.6 & 86.5 & 111.5 & 98.9 & 98.8 & 103.4 & 94.9 \\
Composite & $(3.1)$ & $(6.2)$ & $(2.7)$ & $(6.1)$ & $(5.6)$ & $(5.1)$ & $(4.1)$ & $(5.7)$ \\
scores(SD) & & & & & & & & \\
\hline
\end{tabular}

Conclusion Parental questionnaire using the social-emotional and adapative behaviour items can be used as screening tool to identify high-risk infants requiring speech and physiotherapy intervention. This may allow streamlining of clinics that run the 2 year assessments. This data needs to be validated with a larger cohort.

\section{G167 PROMOTING TECHNOLOGY ENHANCED LEARNING: ASSESSING VIEWS AND EFFICIENCY OF MOBILE PHONES IN CLINICAL PRACTICE}

\begin{abstract}
${ }^{1,2} \mathrm{C}$ Junk, ${ }^{2}$ J Wallace, ${ }^{1} \mathrm{P}$ Mallett, ${ }^{1,2} \mathrm{~A}$ Thompson. ${ }^{1}$ Department of Paediatric Simulation and Education, Royal Belfast Hospital for Sick Children, Belfast, UK; ${ }^{2}$ Department of Paediatrics and Child Health, Royal Belfast Hospital for Sick Children, Belfast, UK
\end{abstract}

\subsection{6/archdischild-2018-rcpch.163}

Aims Technology-enhanced learning, specifically the use of mobile devices by Healthcare professionals has transformed many aspects of clinical practice. ${ }^{1}$

Some healthcare organisations are reluctant to advocate the staff use of mobile phones due to the risks associated with 